\title{
Population Dynamics Through Metapopulation Models: When do Cyclic Patterns Appear?
}

\author{
P. FREDERICO ${ }^{1}$, G.A. CANZIANI ${ }^{2}$ Universidad Nacional del Centro de la \\ Provincia de Buenos Aires, Campus Paraje Arroyo Seco, 7000 Tandil, Argentina.
}

\begin{abstract}
We consider a theoretical ecosystem divided into an infinite number of identical patches and occupied by an ideal species without competitors. The global state of the system is given by a vector in R2 whose coordinates represent the proportion of empty and non-empty patches at time t. The dynamics of the system is described by a non linear Markov chain whose transition matrix depends explicitly on the state of the ecosystem at time t. This type of models were initially constructed for the study of two-species competition, considering constant probabilities of interaction and disturbances. When the probability of disturbance on the processes of colonization and persistence are not constant, complexity makes necessary to understand first the dynamics of one species, before facing the problem of more species. Barradas \& Canziani approached the problem of persistence of one species when the probability of disturbance affecting persistence depends on the proportion of occupied patches and that affecting colonization remains constant. Here we study the symmetric case, i.e. a species is subject with density dependent probability to disturbances affecting colonization, and constant probability of disturbance affecting persistence. We investigate the possibility of existence of periodical solutions when equilibria become unstable, and detect cases in which equilibria always remain stable. Analysis of different cases indicate that given the appropriate conditions all situations may appear: globally stable equilibria, locally stable equilibria, unstable equilibria, and cyclic behavior.
\end{abstract}

\section{Introduction}

Understanding the dynamics of a given ecosystem is one of the central problems in Ecology. That is, knowing at every moment which species are part of it as well as the interactions, both interspecific and intraspecific, within the system. Species coexistence, competition, and diversity are important elements in ecosystem dynamics. The importance of distinguishing local from regional processes when determining diversity patterns has been stressed by Ricklefs [6]. Metapopulation models have been conceived with the purpose of including at least two spatial scales and associating processes occurring at those scales. A family of metapopulation

\footnotetext{
${ }^{1}$ pfederi@exa.unicen.edu.ar

2 canziani@exa.unicen.edu.ar
} 
models has been proposed by Caswell and Cohen in [4] and [5], Barradas and Cohen [3], Barradas, Caswell and Cohen [2]. These are non linear Markov chain models that explicitly include interspecific interaction rates and probabilities of external disturbances.

One advantage of formulating the model as a Markov chain is the fact that variability at patch level can be analyzed, while the system is in equilibrium at a global level. These models were initially built for two competing species, considering constant probabilities of disturbance and constant interaction rates. Barradas and Cohen [3] provided the first rigorous mathematical proof of the coexistence of competing species under external disturbances, creating a mathematical model that explains the existence of fugitive species and the role of disturbances in the increase or decrease of biodiversity. They obtained necessary and sufficient conditions on parameters that guarantee the existence of a non trivial globally stable equilibrium solution. Technical difficulties forced the authors to use constant probabilities of disturbance affecting colonization or persistence. Barradas and Canziani [1] have approached the problem of non constant, density dependent probability of disturbance affecting persistence and constant probability of disturbance affecting colonization by studying first the dynamics of one species. Under these assumptions, more than one non trivial steady state solution may appear, which is not necessarily stable. Moreover, the trivial steady state solution is not always unstable, which means that the species can reach extinction.

Here we will study the behavior of a system with only one species, using the same non linear Markov chain formulation, under the assumption of a density dependent probability of disturbance affecting colonization and a constant probability of disturbance affecting persistence.

\section{Model with Density-Dependent Disturbances}

\subsection{The Model}

We consider an ecosystem occupied by one species and divided into an infinite number of patches, each of which can be either inhabited (state 1) or empty (state $0)$. Let $y_{0}(t)$ and $y_{1}(t)$ denote the proportion of empty and inhabited patches respectively. Changes in the ecosystem are represented by the behavior of a dynamical system in $\Re^{2}$. Nevertheless, the fact that each patch has only two possible states implies that one of the components of fully determines the other:

$$
y_{0}(t)+y_{1}(t)=1 \text {. }
$$

The processes considered here are colonization of empty patches by the species, persistence of the species in an occupied patch, and disturbances affecting both. Colonization is assumed to be proportional to the number of occupied patches in the previous time step and independent of neighborhood effects, hence it is given by the Poisson distribution

$$
C\left(y_{1}\right)=1-\exp \left(-d y_{1}\right)
$$


where $d \geq 0$ denotes the dispersal coefficient of the species.

Disturbances affecting the colonization process are assumed to occur with probability $f\left(y_{1}\right)$, and those affecting persistence in occupied patches with probability $g\left(y_{1}\right)$. This kind of disturbances represent processes of different types, like occupancy-induced predation, fires induced by the abundance of flammable organic material in forests and pastures, etc., all of which are dependent on the density of patch occupancy.

The dynamics of the system is described by a non linear Markov chain

$$
y(t+1)=A_{y(t)} y(t),
$$

where

$$
A_{y(t)}=\left(\begin{array}{cc}
1-\left(1-\exp \left(-d y_{1}\right)\left(1-f\left(y_{1}\right)\right)\right. & g\left(y_{1}\right) \\
\left(1-\exp \left(-d y_{1}\right)\right)\left(1-f\left(y_{1}\right)\right) & 1-g\left(y_{1}\right)
\end{array}\right)
$$

and

$$
y(t)=\left(y_{0}(t), y_{1}(t)\right)^{T} .
$$

Under these same hypotheses, Barradas and Cohen [3] considered the probabilities of disturbance to be constant, while Barradas and Canziani [1] considered a constant function $f\left(y_{1}\right)$ and several different biologically meaningful functions $g\left(y_{1}\right)$ for the probability of disturbance affecting persistence. In both cases different types of steady states were found, depending on given conditions.

In order to understand the dynamics of the system, we first search for steady states. Note that constant $y(t)$ does not exclude changes in the occupancy of particular patches, but reflects only that the proportion of occupancy remains constant.

Because of the fact that $y_{0}+y_{1}=1$, a fixed point of (2.1) satisfies two equivalent equations derived from $A_{y} y=y$, that can be written as

$$
H(y)=(1-\exp (-d y))(1-f(y))(1-y)+(1-g(y)) y=y,
$$

where $y=y_{1}$.

Finding solutions of (2.3) is equivalent to finding zeros of $h(y)=H(y)-y$. Since $H(0)=0$ and $H(1) \leq 1$, one way of assuming the existence of at least one positive solution of $(2.3)$ is to ask for $H(0)>1$. In such a case the condition on $H(y)$ yields

$$
d>\frac{g(0)}{1-f(0)}
$$

provided $f(0) \neq 1$. It is clear that the case $f(0)=1$ does not make sense in real biological settings. This condition is analogous to condition 0.5 in Barradas and Cohen [3] and condition 4 in Barradas and Canziani [1].

\subsection{Density Dependent Probability of Disturbance During Colonization}

Given the general form of the probability functions $f(y)$ and $g(y)$, it is possible that more than one non trivial steady state can be found. Here we will consider $g(y)$ 
to be constant, say $g(y)=p$, and $f(y)$ will take the form of different biologically meaningful functions.

Note that $H(y)$ can be split into two terms, one involving $f(y)$ and the other involving $g(y)$, in a way that can simplify the analysis of existence and behavior of steady states. Let

$$
\varphi(y)=(1-\exp (-d y))(1-f(y))(1-y)
$$

and let

$$
G(y)=p y
$$

Note that $\varphi(0)=G(0)$ and $\varphi(1)=0$, and that solving

$$
H(y)=y
$$

is equivalent to solving the equation

$$
\varphi(y)=G(y) .
$$

One sufficient condition for the existence of a solution is that

$$
\varphi^{\prime}(0)>G^{\prime}(0)=p
$$

from which condition (2.4) can be obtained.

\subsection{Stability of Steady State Solutions of the Model}

When a steady state solution is found, its stability can be determined following the general conditions for discrete dynamic systems. A fixed point $y^{*}$ of $H(y)$ is locally stable and $\left(1-y^{*}, y^{*}\right)^{T}$ is a locally stable solution for $(2.3)$ if

$$
-1<H^{\prime}\left(y^{*}\right)<1
$$

In our case

$$
\begin{aligned}
H^{\prime}(y)= & d \exp (-d y)(1-f(y))(1-y) \\
& +(1-\exp (-d y))\left[-f^{\prime}(y)(1-y)-1+f(y)\right]-p .
\end{aligned}
$$

On the other hand, when $y^{*}$ is such that $H\left(y^{*}\right)=y^{*}$, we obtain

$$
\exp \left(-d y^{*}\right)=1-\frac{p y^{*}}{\left(1-f\left(y^{*}\right)\right)\left(1-y^{*}\right)} .
$$

Replacing these expressions in equation (2.6) we obtain

$$
-1<-p\left\{y^{*}\left[d+\frac{f^{\prime}\left(y^{*}\right)}{1-f\left(y^{*}\right)}+\frac{1}{1-y^{*}}\right]+1\right\}+d\left(1-y^{*}\right)\left(1-f\left(y^{*}\right)\right)<1 .
$$

Instead of considering with the equation $H(y)=y$, we can consider $\varphi(y)=G(y)$, and search for an equivalent condition involving $\varphi^{\prime}(y)$. 
Criterion 1 Stability Condition. A solution $y^{*}$ of $\varphi(y)=G(y)$ is locally stable if

$$
p-2<\varphi^{\prime}\left(y^{*}\right)<p .
$$

Proof. If we write $H(y)=\varphi(y)+(1-p) y$ then $H^{\prime}(y)=\varphi^{\prime}(y)-p+1$ in which case condition (2.5) is equivalent to $-1<\varphi^{\prime}\left(y^{*}\right)-p+1<1$.

Hence we obtain $p-2<\varphi^{\prime}\left(y^{*}\right)<p$.

Criterion 2 Instability Condition. If a steady state $y^{*}$ corresponds to an intersection of curves $\varphi(y)$ and $G(y)$ such that $\varphi(y)<G(y)$ when $y<y^{*}$, then $y^{*}$ is an unstable equilibrium.

Proof. Given $\varepsilon>0$, we can write

$$
\frac{\varphi\left(y^{*}+\varepsilon\right)-\varphi\left(y^{*}\right)}{\varepsilon}>\frac{p\left(y^{*}+\varepsilon\right)-p y^{*}}{\varepsilon} .
$$

Taking limits on both sides as $\varepsilon \rightarrow 0$, we get

$$
\varphi^{\prime}\left(y^{*}\right) \geq p .
$$

By the previous criterion $y^{*}$ is unstable.

\section{Particular Familes of Functions $f(y)$}

When $f(y)$ is no longer constant, the function $\varphi(y)$ can not be expected to be concave, a fact that partially simplified the analysis in Barradas and Canziani [1]. In order to appreciate the richness of behavior of the system, it is then necessary to consider different probability functions $f(y)$.

Graphically, the solutions of the equation $\varphi(y)=G(y)$ are the points of intersection of the curve $\varphi(y)$ with the straight line $G(y)$. Since the line $G(y)$ passes through the origin, it is fundamental to analyze the concavity of $\varphi(y)$ in its neighborhood in order to determine the number of non trivial steady states. In particular, it is important to analyze the concavity of $\varphi(y)$ between the origin and the point where this function attains its maximum over the interval $[0,1]$ as can be seen in the examples.

\subsection{Case I}

We consider the case in which the probability of disturbance occurring during colonization is high at low occupation and decreases as the occupation of patches increases. This could be the case in a region such as the Patagonia. When the density of the vegetation cover is low, the winds can hinder the germination and rooting of seeds, but the effect is diminished as the density of grown plants increases. 
This situation can be described using a family of functions of the form

$$
f(y)=1-\frac{y^{2}}{k+y^{2}},
$$

where $k$ is a positive constant. Then $0<f(y)<1$.

We can then compute the derivatives $\varphi^{\prime}(y)$ and $\varphi^{\prime \prime}(y)$ and observe that both $\varphi^{\prime}(0)=0$ and $\left.\varphi^{\prime \prime} 0\right)=0$. In order to produce a formal analysis of the concavity of $\varphi(y)$ over the interval $[0,1]$, it is necessary to observe the sign of the second derivative and obtain some relationship involving parameters $d$ and $k$, and the variable $y$. It can be easily seen that the expressions of derivatives are rather intractable. Nevertheless we can consider $\varphi(y)$ as the product of two functions:

$$
\varphi(y)=\bar{\varphi}(y) \bar{f}(y)
$$

where $\bar{\varphi}(y)=(1-\exp (-d y))(1-y)$ verifying $\bar{\varphi}(0)=\bar{\varphi}(1)=0$. Moreover, $0 \leq \bar{\varphi}(y) \leq 1-y, \quad \forall y \in[0,1]$.

The first derivative of this function is

$$
\bar{\varphi}^{\prime}(y)=(d-d y+1) \exp (-d y)-1
$$

verifying $\bar{\varphi}^{\prime}(0)=d>0$ and $\bar{\varphi}^{\prime}(1)=\exp (-d)-1<0$.

From the analysis of the second derivative we obtain the following condition for the concavity of the function:

$$
\bar{\varphi}^{\prime \prime}(y)=-d \exp (-d y)(-d y+d+2)<0 \quad \Leftrightarrow \quad-d y+d+2<0
$$

so that it is necessary that $y<\frac{d+2}{d}$, a condition that is verified $\forall y \in[0,1]$. Then we can conclude that $\bar{\varphi}(y)$ is concave on the interval $[0,1]$.

Let us now consider $\bar{f}(y)=\frac{y^{2}}{k+y^{2}}$, and such that $\bar{f}(0)=0$. This is an increasing function of $y$ for

$$
\bar{f}^{\prime}(y)=\frac{y^{2}}{k+y^{2}}>0 \quad \forall y \in[0,1] .
$$

It is also a convex function on the interval $[0, \sqrt[2]{k}]$, for the second derivative satisfies

$$
\bar{f}^{\prime \prime}(y)=\frac{2 k\left(k-y^{2}\right)}{k+y^{2}}>0 \Longleftrightarrow|y|<\sqrt{k} .
$$

If we express $\bar{\varphi}^{\prime}(y)$ in terms of $\bar{\varphi}(y), \bar{f}(y)$, and their derivatives, we obtain the expression:

$$
\bar{\varphi}^{\prime \prime}(y)=\bar{\varphi}^{\prime \prime}(y) \bar{f}(y)+2 \bar{\varphi}^{\prime}(y) \bar{f}^{\prime}(y)+\bar{\varphi}(y) \bar{f}^{\prime \prime}(y)
$$

in which the first term is negative on $[0,1]$, the second is positive in a neighborhood of zero, and the third one is positive for $y \in[0, \sqrt[2]{k}]$. Therefore, in order to prove that $\bar{\varphi}(y)$ is convex in a neighborhood of zero, it is sufficient to prove that:

$$
-\bar{\varphi}^{\prime \prime}(y) \bar{f}(y)<2 \bar{\varphi}^{\prime}(y) \bar{f}^{\prime}(y)
$$


or more precisely

$$
d \exp (-d y)(-d y+d+2) \frac{y^{2}}{k+y^{2}}<2[\exp (-d y)(d-d y+1)-1] \frac{2 k y}{k+y^{2}}
$$

From this inequality we obtain

$$
\underbrace{y^{2} \frac{d^{2}}{4 k}+y\left(\frac{-d^{2}-4 k d-2 d}{4 k}\right)+d+1}_{A(y)}>\underbrace{\exp (d y)}_{B(y)} .
$$

Both functions, $A(y)$ and $B(y)$, are continuous, and $d+1=A(0)>B(0)=1$, which implies that $\exists \varepsilon>0$ such that $A(y)>B(y) \quad \forall y \in[0, \varepsilon)$. This proves that $\varphi(y)$ is convex in a neighborhood of zero, and then concave. This was also observed plotting the function for a wide range of values of $d$ and $k$.

This is one of those examples where the sufficient condition (2.4) is not verified, yet there are equilibrium solutions depending on the values of $p, d$, and $y$.

\subsubsection{Criteria for the existence of steady states}

Let us consider the graph of $\varphi(y)$ over the interval $[0,1]$ and a vertical line passing through the origin. If the line is tilted towards the right using the origin as a pivot, there is a point $\left(y_{0}, \varphi\left(y_{0}\right)\right)$ at which the line will tangentially touch the curve. In other words, if $T(y)$ is the tangent to the curve passing through the origin, it will satisfy the equation:

$$
T(y)-\varphi\left(y_{0}\right)=\varphi^{\prime}\left(y_{0}\right)\left(y-y_{0}\right)
$$

and therefore $T(y)=\varphi^{\prime}\left(y_{0}\right) y+\varphi\left(y_{0}\right)-\varphi^{\prime}\left(y_{0}\right) y_{0}$. Note that this condition is trivially verified for $y=0$ since $\varphi^{\prime}(0)=0$.

If now we focus on this point $\left(y_{0}, \varphi\left(y_{0}\right)\right)$ and on the value of the derivative $\varphi^{\prime}\left(y_{0}\right)$, we can formulate the following criteria, depending on the disturbance probability $p$ (Fig.1):

i. Non existence of non trivial steady states if $p>\varphi^{\prime}\left(y_{0}\right)$

ii. Existence of one non trivial steady state if $p=\varphi^{\prime}\left(y_{0}\right)$

iii. Existence of two non trivial steady states if $p<\varphi^{\prime}\left(y_{0}\right)$.

Let $p, d$ and $k$ be such that there exists a steady state. Let $k$ be fixed, then decreasing $p$ and increasing $d$, or inversely, we can obtain two steady states that shift one towards $y=0$ and the other towards $y=1$ (Fig.1). When $p$ is fixed, an increase in $d$ and a decrease in $k$ produces a shift of two steady states towards the trivial ones. 


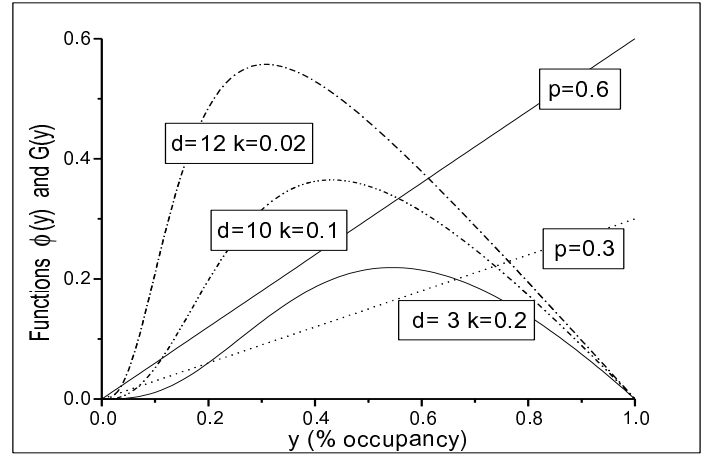

Figure 1: Illustrates Case I

\subsubsection{Stability of steady states}

When the probability of disturbance affecting persistence is strictly positive, that is $p>0$, we can state that:

i. The trivial steady state $y^{*}=0$ is always stable since $\varphi^{\prime}(0)=0<p$, satisfies condition (2.9). This means that, independently of the value of $p>0$, when the probability of occurring a disturbance that affects colonization is high at low patch occupancy, the species can not persist.

ii. When the only non trivial steady state is $y^{*}=y_{0}$, it is unstable since $\varphi^{\prime}(0)=p$. iii. When conditions are such that two non trivial steady states, $y_{*}<y^{*}$, are obtained, then $y_{*}$ will be unstable for the Instability Criterion is satisfied. The stability of $y^{*}$ can not be determined unless $d$ and $k$ are known explicitly. Since only cases where $p<1$ are meaningful, it is enough that the condition $\varphi^{\prime}\left(y^{*}\right) \geq-1$ is verified to assure stability whenever $y^{*}$ is at the right of the point where $\varphi(y)$ attains its maximum, independently of the value of $p$. This can be easily verified graphically. When $y^{*}$ is at the left of the point where $\varphi(y)$ attains its maximum, it is because $p$ has a high value, in which case $\varphi^{\prime}\left(y^{*}\right)$ is in fact very small. Then the condition $\varphi^{\prime}\left(y^{*}\right)<p$ is satisfied.

When the probability of disturbance affecting persistence is null, the only steady states are the trivial ones, $y_{*}=0$ and $y^{*}=1$. Under this condition $y_{*}=0$, representing total emptiness, is an unstable equilibrium since $\varphi^{\prime}(0)=p=0$. On the other hand $y^{*}=1$, representing total occupancy, is a globally stable equilibrium since condition (2.9) is verified for $\varphi^{\prime}(1)$. When the probability of a disturbance affecting persistence is null, no matter how high the probability of disturbance during colonization, the species will eventually occupy the whole territory. When a patch is colonized, the species will persist forever and will continue colonizing other patches from this one. 


\subsection{Case II}

Let us consider the case where the probability of a disturbance during colonization increases as the proportion of occupied patches increases. This could represent situations where competition, both intraspecific and interspecific, is important. For instance, if the density of trees in a certain region is high, the shadow of the canopy affects photosynthesis and hence reduces the chances of the offsprings of growing and prospering. Another example could be that of a predator-prey system, where an increase of prey occupancy of the territory attracts the predator species and produces an increase in the probability of disturbance affecting the process of colonization by the prey species. The probability function can have the form:

$$
f(y)=\alpha y^{\beta},
$$

with $0<\alpha \leq 1, \beta>0$.

The analysis of the concavity of $\varphi(y)$ is not trivial. Once more we can consider $\varphi(y)$ as the product of two functions

$$
\varphi(y)=\bar{\varphi}(y) \bar{f}(y),
$$

when $\bar{\varphi}$ is as before, and $\bar{f}(y)=1-\alpha y^{\beta}$. In order to study the latter we need to consider four cases, depending on the value of $\beta$ and its role in the derivatives of the function:

(a) $\beta \geq 2$. Given this condition, we have: $\bar{f}^{\prime}(y)=-\beta \alpha y^{\beta-1} \leq 0 \quad \forall y \in[0,1]$ and $\bar{f}^{\prime \prime}(y)=-\beta(\beta-1) \alpha y^{\beta-2}<0 \quad \forall y \in(0,1]$. We have $\bar{f}^{\prime \prime}(0)=0$, which implies that $\bar{f}(y)$ is concave over the interval $[0,1]$. Under these conditions, and recalling that $\bar{\varphi}(y)$ is also concave on $[0,1]$, we can affirm that $\varphi(y)$ is concave on $\left[0, y_{m}\right]$, where $y_{m}$ is the maximun of $\varphi(y)$ on $[0,1]$.

(b) $1<\beta<2$. Here we also have $\bar{f}^{\prime}(y)=-\beta \alpha y^{\beta-1} \leq 0 \quad \forall y \in[0,1]$. The difference with the previous case is that the second derivative is not defined at $y=0$. Nevertheless we can see that

$$
\bar{f}^{\prime \prime}(y)=-\beta(\beta-1) \alpha y^{\beta-2}<0, \quad \forall y \in(0,1],
$$

so that when taking limits as $y \longrightarrow 0$ we obtain: $\lim _{y \rightarrow 0} \bar{f}^{\prime \prime}(y)=-\infty$. This implies that $\bar{f}(y)$ is concave on the interval $[0,1]$. Then, as before, $\varphi(y)$ is concave on $\left[0, y_{m}\right]$.

(c) $\beta=1$. Here we have a straight line $\bar{f}(y)=1-\alpha y$ so that, when multiplied by the concave function $\bar{\varphi}(y)$, we obtain a concave function on the interval $\left[0, y_{m}\right)$.

(d) $0<\beta<1$. In this case we have

$$
\bar{f}^{\prime}(y)=-\beta \alpha y^{\beta-1}<0, \quad \forall y \in(0,1],
$$

hence by taking limits as $y \rightarrow 0$ we obtain $\lim _{y \rightarrow 0} \bar{f}^{\prime}(y)=-\infty$. 
Differently from situations (a) and (b), here the second derivative is

$$
\bar{f}^{\prime \prime}(y)=-\beta(\beta-1) \alpha y^{\beta-2}>0, \forall y \in(0,1],
$$

so that $\lim _{y \rightarrow 0} \bar{f}^{\prime \prime}(y)=+\infty$ which means that $\bar{f}(y)$ is convex on the interval $\left[0, y_{m}\right]$.

Under these conditions it is not possible to ascertain the concavity of $\varphi(y)$ straightforwardly as in the previous cases. Since the first and second derivatives of $\bar{f}(y)$ are not defined at $y=0$, it is not possible either to deduce the sign of the second derivative $\varphi^{\prime \prime}(y)$ as in Case I. Nevertheless we can affirm that $\varphi(y)$ is concave in a neighborhood of 0 for

$$
\lim _{y \rightarrow 0^{+}} \varphi^{\prime \prime}(y)=-\infty .
$$

Then the only possibility for knowing the general behavior of the function $\varphi(y)$ over the interval $\left[0, y_{m}\right]$ is to plot it for a wide range of values of parameters $d$ and $\beta$. It is also important to plot the second derivative too, for there can be values of the parameters for which inflection points appear but are imperceptible on the graph. We can conclude that the function $\varphi(y)$ is concave in a neighborhood of 0 and, moreover, the inflection point is found beyond the point where the function reaches its maximum.

\subsubsection{Criteria for the existence of steady states}

i. If $\beta \geq 1$, then $\varphi^{\prime}(0)=d$ and we get from (a), (b), and (c) that $\varphi(y)$ is concave over $\left[0, y_{m}\right]$. Therefore the existence of a non trivial steady state depends on the value of $d$, as established by condition (2.4) (Fig. 2). If $d<p$ there is no non trivial steady state. If instead $d>p$, there exists a non trivial steady state and it will be unique due to the concavity of $\varphi(y)$ over the whole interval $\left[0, y_{m}\right]$.

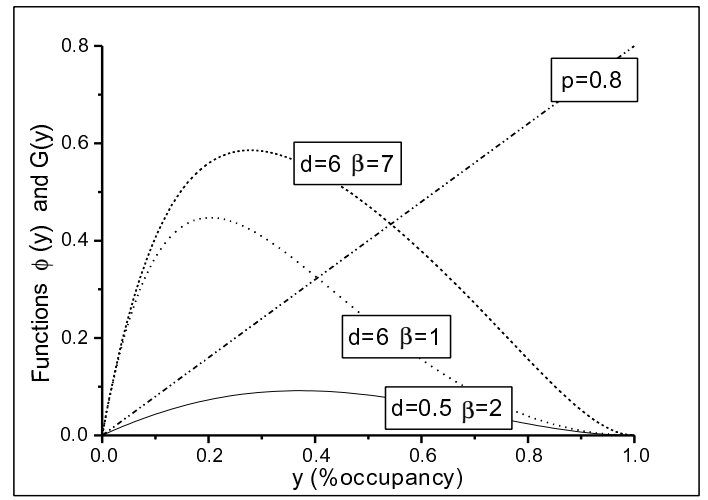

Figure 2: Illustrates Case II when $\beta \geq 1, \alpha=1$

ii. If $\beta<1$, then $\lim _{y \rightarrow 0^{+}} \varphi^{\prime}(y)=+\infty$. This guarantees the existence of a non trivial steady state for any value of the other parameters. This steady state is also 
unique for $\varphi(y)$ does not change its concavity until after it reaches its maximum value (Fig. 3).

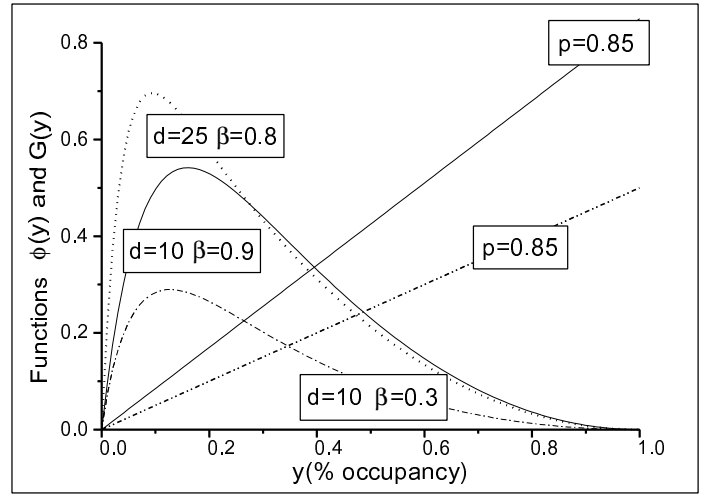

Figure 3: Illustrates Case II when $\beta<1, \alpha=1$

In both cases the non trivial equilibrium moves towards the trivial one either by increasing $d$ or $\beta$, or by decreasing $p$.

\subsubsection{Stability of the steady states}

If the probability $p$ of a disturbance affecting persistence is positive, we obtain the following results depending on whether $\beta \geq 1$ or $\beta<1$ :

When $\beta \geq 1$ the steady state $y_{*}=0$ is stable for $d<p$ and unstable in the other cases. This means that in order to be successful in the process of colonization it is not sufficient for the species that the probability of perturbation be low but also needs to have a capability for dispersion such that it can overcome the effect of disturbances on persistence. A single non trivial steady state $y^{*}$ exists only if $d>p$. Its stability depends on the values of the other parameters in $f(y)$ and of $p$.

When $\beta<1$ the steady state $y_{*}=0$ is always unstable independently of the capability of dispersion of the species and the probability of disturbances affecting persistence. As before, the stability of the non trivial steady state $y^{*}$ depends on the relative value of the parameters, with a greater sensitivity towards the value of $p$.

Example. Considering the following values for the parameters $d=10, \alpha=1$, $\beta=0.9$, and $p=0.7$, the steady states are:

$y_{*}=0$, with $\varphi^{\prime}(0)=+\infty$, is unstable;

$y^{*}=0.42896030$, with $H^{\prime}\left(y^{*}\right)=-0.7357575$, is stable.

If the value of $p$ is changed to $p=0.95$, then the steady states become: $y^{*}=$ 0.3757851 , with $H^{\prime}\left(y^{*}\right)=-1.041727$, is in this case unstable.

This means that when $p<p_{0}$ for a certain $p_{0}$, then $y^{*}$ is stable but looses the stability when $p>p_{0}$. In the latter case there exist two consecutive unstable steady 
states, which give rise to cyclic behavior (See Fig. 5).

When the probability of a disturbance affecting persistence $p=0$, the trivial steady state $y_{*}=0$ is unstable for any positive value of $\beta$. In this case the species is under totally favorable conditions, because at low patch occupancy the probability of a disturbance affecting colonization is low and the probability of a disturbance affecting persistence is null. Even when the probability of disturbance affecting colonization increases with density, the species is not affected for once a patch is colonized it is never lost. The other trivial steady state $y^{*}=1$ is globally stable. Condition $(2.9)$ is verified since $\varphi^{\prime}(1)=(1-\alpha) \exp (-d)+\alpha-1$ satisfies $-1<\alpha<(1-\alpha) \exp (-d)+\alpha-1<0$.

\subsubsection{Periodic Solutions}

Let us consider $f(y)=\alpha y^{\beta}$ and take the parameters to be $\alpha=1, \beta=0.9, d=10$, and different values for $p$. When we plot the iterated function $H(H(y))$ together with the identity for several values of $p$, as specified in the figure, we can observe in each case one intersection of the two curves, corresponding to the solutions of $H(y)=y$. It is important to note how the plots change as the value of $p$ increases (Fig.4). The plot for $p=0.98$ shows three intersection points, which implies the existence of period-two solutions. The stability of period-two solutions is determined by evaluating the slope at each intersection point.

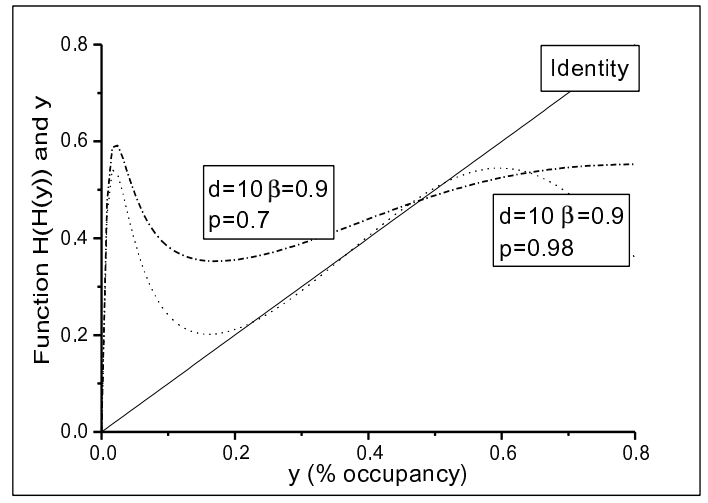

Figure 4: Emergence of cycles as equilibrium becomes unstable

In this case the existence of periodic solutions can be interpreted as follows. When the occupancy level is low, the species finds favorable conditions for colonization independently of the probability of disturbance affecting persistence, for an emptied patch is rapidly colonized again. Then the density of occupied patches grows fast. As high levels of occupancy are reached, the probability of disturbance affecting colonization increases and couples its effects with the probability of disturbance affecting persistence in such a way that an emptied patch has a low 
probability of being successfully colonized again. This leads to a decrease in the density of occupied patches until the system returns to a situation favoring colonization. The system oscillates (Fig. 5) between two states: one at low and the other at high level of patch occupancy.

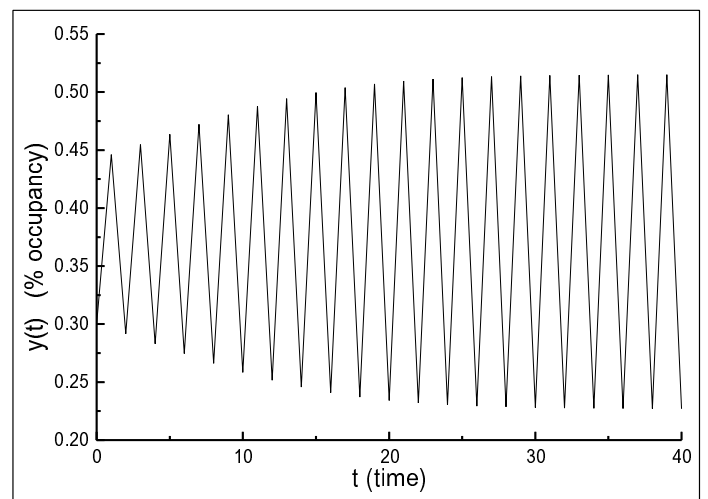

Figure 5: Cyclic behaibor with parameters as in Example

\subsection{Case III}

Let us consider now a case in which the probability of a disturbance affecting colonization is high at both low and high patch occupancy while at intermediate densities it becomes low. As an example we can consider a plant species that can not survive if too exposed, but its growth is hindered by intraspecific competition (shadow).

We can represent such situation using the function

$$
f(y)=\alpha \frac{\cos (2 \pi y)+1}{2}
$$

with $0<\alpha \leq 1$.

As before function $\varphi(y)$ obtained for this probability function is unimodal. In this case the analysis of $\varphi(y)$ and the solutions of the equation $\varphi(y)=G(y)$ are based on geometric methods for the analytic expressions are intractable. For a given value of parameter $d$, there exists a point $\alpha_{0} \in(0,1)$ such that one of the following three is valid for $\varphi(y)$, considering $y_{m}=y_{m}(p, d)$ to be the point where $\varphi(y)$ reaches its maximum.

\subsubsection{Criteria for the existence of steady states}

i. When $\alpha \in\left(0, \alpha_{0}\right)$, the function $\varphi(y)$ is concave on the interval $\left[0, y_{m}\right)$ and this implies the existence of a non trivial steady state only if $\varphi^{\prime}(0)>p$. 
ii. When $\alpha \in\left(\alpha_{0}, 1\right)$, the function $\varphi(y)$ exhibits two inflection points $y_{0}$ and $y_{1} \in\left(0, y_{m}\right), y_{0}<y_{1}$, such that $\varphi(y)$ is concave on $\left[0, y_{0}\right)$, convex on $\left(y_{0}, y_{1}\right)$, and concave again on $\left(y_{1}, y_{m}\right)$. These changes in the concavity of the function before reaching its peak value give rise to the possibility of more than one intersection with the linear function $G(y)$. Hence up to three non trivial steady states can be found.

iii. When $\alpha=1$, the function $\varphi(y)$ shows a unique inflection point on the interval $\left[0, y_{m}\right)$, being first convex and then concave. This is a function that behaves as the one in Case I, so that the conditions for the existence of non trivial steady states are the same.

Here situation ii., even if mathematically interesting, is not applicable to biologically meaningful situations since the changes in concavity are essentially imperceptible. When adjusting a model to field data, only situations i. and iii. will be observed. In situation i., where only one non trivial equilibrium is found, an increase in $d$ or a decrease in $p$ will shift the equilibrium towards $y=1$ for any fixed value of $\alpha$. The same behavior is observed when $\alpha$ decreases for this implies a lower probability of disturbance at both high and low patch occupancy.

\subsubsection{Stability of the steady states}

In situation i., that is for $\alpha \in\left(0, \alpha_{0}\right)$, the probability of a disturbance affecting colonization is low at low patch occupancy, and the behavior is similar to that of Case II with $\beta \leq 1$. The trivial steady state $y_{*}=0$ is unstable when $\varphi^{\prime}(0)=$ $(1-\alpha) d>p$. When the value of $\alpha$ is close to 0 , the behavior is similar to the one for $\beta \leq 1$ in Case II. If there exists a non trivial equilibrium, it can be either stable or unstable, in which case period two solutions can be obtained.

In situation iii., that is for $\alpha=1$, the probability of a disturbance affecting colonization is very high when densities are close to the endpoints of the interval $[0,1]$. Hence the stability of the trivial steady state $y_{*}=0$, and of the first non trivial one in case it exists, is given as in Case I. There is a fundamental difference with Case I when there exists a second non trivial equilibrium, which relates to the high probability of disturbance at high patch occupancy, since it can become unstable when the probability of disturbance affecting persistence is also high. In the case where the second non trivial steady state is unstable, period two solutions appear.

\section{Conclusions}

We can observe in this study how the fact of introducing a density dependent probability of disturbances affecting the process of colonization opens the door to a variety of behaviors, including the possibility of cycles. The number and stability of the steady states in the level of occupancy of the ecosystem vary depending on the nature of the probability function $f(y)$.

When the probability of disturbance affecting colonization is high at low patch occupancy, then the trivial steady state $y_{*}$ is always stable. A high probability of 
disturbance at low patch occupancy puts the species in a most unfavorable situation facilitating extinction, as seen in Case I or Case III when $\alpha \rightarrow 1$.

High values of the dispersion coefficient $d$ enhance the appearance of non trivial equilibria, as seen in Case I, and induce the instability of the trivial equilibrium $y_{*}$, as in Case II (when $\beta \leq 1$ ) and in Case III.

When $p$ the probability of disturbance affecting persistence is not too high, a low probability of disturbance affecting colonization at low patch occupancy results in general in a steady state with high percentage of occupied patches. Even when $p$ is high, periodic cycles may appear, but there is no risk of extinction.

The metapopulation approach is not much used yet in analysis of population dynamics but some publications show that it could be a very promising one (Valverde and Silvertown [7]).

\section{References}

[1] I. Barradas and G.A. Canziani, A study of persistence under density dependent disturbances, in Anales de la VII RPIC, vol II pp.797-802, San Juan, Argentina, 1997.

[2] I. Barradas, H. Caswell and J.E. Cohen, Competing during colonization vs. competition in disturbed environments: a metapopulation approach. Bull. Math. Biol., 58(6) (1996), 1187-1207.

[3] I. Barradas and J.E. Cohen, Competition during colonization vs. Competition after colonization in disturbed environments: a metapopulation approach. Bull. Math. Biol., 58 No.6 (1994), 1187-1207.

[4] H. Caswell, J.E. Cohen, "Communities in patchy environments: a model of disturbance, competition, and heterogeneity". Ecological Heterogeneity, Kolasa, J. Springer-Verlag, Berlin, Heidelberg, New York, 1990.

[5] H. Caswell, J. E. Cohen, Disturbance and diversity in metapopulations. Biol. J. Linn. Soc., 42 (1991), 193-218.

[6] R. E. Ricklefs, Community diversity: relative roles of local and regional processes. Science, 235 (1987), 167-171.

[7] T. Valverde, J. Silvertown, A metapopulation model for Primula vulgaris, a temperate forest understorey herb. Journal of Ecology, 84 (1996). 
